\title{
LOCALLY UNIFORMLY ROTUND NORMS AND MARKUSCHEVICH BASES
}

\author{
A. C. YORKE \\ (Received 10 January 1977; revised 10 April 1978) \\ Communicated by E. Strzelecki
}

\begin{abstract}
If a Banach space $E$ admits a Markuschevich basis, then $E$ can be renormed to be locally uniformly rotund. When the coefficient space of the basis is 1-norming, and this norm is very smooth, $E$ is weakly compactly generated.
\end{abstract}

Subject classification (Amer. Math. Soc. (MOS) 1970): 46 B 99.

\section{Introduction}

Dyer (1969), page 55, has shown that if $E$ admits a Markuschevich basis, then there is a continuous one-to-one linear operator which maps $E$ into some $c_{0}(\Gamma)$. Hence, by a result of Klee (1953), page $56, E$ can be equivalently renormed to be rotund. It will be shown here that if $E$ admits a Markuschevich basis, then $E$ can be renormed with a locally uniformly rotund norm which is $\sigma(E, Y)$ lower semi-continuous, where $Y$ is the coefficient space of the basis. This norm is an equivalent norm for $E$ if the coefficient space is norming (Zizler and John (1974b)). If the coefficient space is 1-norming, and this norm is very smooth, then the Markuschevich basis for $E$ is shrinking, so $E$ is weakly compactly generated. This improves a result of Zizler and John (1974b), page 687.

This paper is based on part of a doctoral thesis submitted to the University of Newcastle under the supervision of Associate Professor J. R. Giles.

\section{Notation and definitions}

Let $E$ be a real Banach space, $E^{*}$ its dual, and $\hat{E}$ the canonical embedding of 
$E$ in $E^{* *}$. The unit spheres of $E$ and $E^{*}$ will be denoted by $S(E)$ and $S\left(E^{*}\right)$, respectively. The term 'subspace' will always mean norm closed linear subspace. The density character of a subspace $Y \subset E$, denoted dens $Y$, is the minimum cardinality of a norm dense subset of $Y$. The weak-* density character of a subspace $F \subset E^{*}$, denoted $\sigma\left(E^{*}, E\right)$ dens $F$, is the minimum cardinality of a weak- ${ }^{*}$ dense subset of $F$. The norm closed linear span of a set $A \subset E$ will be denoted by $\overline{\mathrm{sp}} A$.

The set $\left\{\left(x_{i}, f_{i}\right): i \in I\right\}$ contained in $E \times E^{*}$ is said to be a Markuschevich basis (M-basis) for $E$ if

(1) $\left\{\left(x_{i}, f_{i}\right)\right\}$ is biorthogonal; that is, $f_{i}\left(x_{i}\right)=1$, while $f_{i}\left(x_{j}\right)=0$ if $i \neq j$ for all $i \in I$.

(2) $\left\{x_{i}\right\}$ is fundamental in $E$; that is, $\overline{\operatorname{sp}}\left\{x_{i}\right\}=E$.

(3) $\left\{f_{i}\right\}$ is total over $E$; that is, $\bigcap_{i \in I} f^{-1}(0)=\{0\}$.

The subspace $\overline{\mathrm{sp}}\left\{f_{i}\right\}$ of $E^{*}$ is called the coefficient space of the $M$-basis.An $M$-basis is shrinking if $\overline{\operatorname{sp}}\left\{f_{i}\right\}=E^{*}$; boundedly complete if whenever $\left\{y_{\delta}: \delta \in D\right\}$ is a bounded net in $E$ with the property that $\lim _{\delta} f_{i}\left(y_{\delta}\right)$ exists for each $i \in I$, there is an $x \in E$ such that $\lim _{\delta} f_{i}\left(y_{\delta}\right)=f_{i}(x)$ for each $i$.

For any subspace $Y \subset E^{*},\|x\|_{Y}=\sup \{|f(x)|: f \in S(Y)\}$ defineds a semi-norm on $E$. If $Y$ is total over $E$, then $\|\cdot\|_{Y}$ is norm. In general, $\|x\|_{Y} \leqslant\|x\|$ for all $x \in E$; that is, the $\|\cdot\|_{Y}$ topology is a weaker topology than the $\|\cdot\|$ topology. The space $Y$ is said to be norming if the $\|\cdot\|$ and $\|\cdot\|_{Y}$ topologies are equivalent. $Y$ is 1-normingif $\|x\|=\|x\|_{r}$ for every $x \in E$.

$E$ is locally uniformly rotund (LUR) at $x \in S(E)$ if every sequence (or net) $\left\{x_{n}\right\}$ in $S(E)$ with $\left\|x_{n}+x\right\| \rightarrow 2$, has $\left\|x_{n}-x\right\| \rightarrow 0$. $E$ is LUR if it is LUR at every $x \in S(E)$.

The set valued mapping $D_{E}$ of $E$ into $2^{E^{*}}$ which assigns to each $x \in E$ the $\left\{f \in E^{*}: f(x)=\|f\|\|x\|\right.$ and $\left.\|x\|=\|f\|\right\}$ is called the duality mapping. The mapping $x \rightarrow f_{x}$ which sends each $x \in S(E)$ to an $f_{x} \in D_{E}(x)$, and has the property that, for $\lambda>0, f_{\lambda x}=\lambda f_{\mathrm{x}}$ is called a support mapping on $E$. The norm of $E$ is said to be very smooth if every support mapping $x \rightarrow f_{x}$ on $E$ is continuous when $E$ has the norm topology and $E^{*}$ has the $\sigma\left(E^{*}, E^{* *}\right)$ topology (Giles (1975), page 72). The norm of $E$ is Fréchet differentiable ( $F$-differentiable) if every support mapping on $E$ is continuous when both $E$ and $E^{*}$ have the norm topology (see Giles (1971), page $107)$. When the norm of $E$ is very smooth ( $F$-differentiable), the space $E$ is said to be a very smooth ( $F$-differentiable) space.

If $E$ contains a $\sigma\left(E, E^{*}\right)$ compact fundamental subset, then $E$ is weakly compactly generated $(W C G)$.

\section{Main result and applications}

This section will state the Main Result and give several applications. The proof of the Main Result will be given in the next section. 
MAIN ResUlt Let $E$ admit an $M$-basis $\left\{\left(x_{i}, f_{i}\right): i \in I\right\}$ with coefficient space $Y$. Then $E$ can be renormed with a LUR norm which is $\sigma(E, Y)$ lower semi-continuous.

Corollary 1. (Zizler and John (1974b)) Let E admit an M-basis with a norming coefficient space. Then $E$ can be equivalently renormed to be LUR.

COROLlary 2. Let $E$ admit a boundedly complete $M$-basis. Then $E$ can be equivalently renormed to be LUR.

Proof. If the $M$-basis is boundedly complete, then the coefficient space is norming (Johnson (1970a), page 175).

Corollary 3. Let $E^{*}$ admit an $M$-basis $\left\{\left(f_{i}, F_{i}\right): i \in I\right\}$ with the property that its coefficient space $Y$ is contained in $\hat{E}$. Then $E^{*}$ can be renormed with a LUR dual norm.

Proof. Since $\left\{F_{i}: i \in I\right\}$ is total over $E^{*}, \operatorname{sp}\left\{F_{i}\right\}$ is $\sigma\left(E^{* *}, E^{*}\right)$ dense in $E^{* *}$. If $Y \subset \hat{E}$, then each $F_{i}=\hat{x}_{i}$ for some $x_{i} \in E$. Thus $\operatorname{sp}\left\{x_{i}\right\}$ is $\sigma\left(E, E^{*}\right)$ dense in $E$, hence $\overline{\mathrm{sp}}\left\{x_{i}\right\}=E$. Now by the Main Result, $E^{*}$ admits a LUR norm which is $\sigma\left(E^{*}, E\right)$ lower semi-continuous.

Corollary 4. Let $E^{*}$ be as in Corollary 3. Then E is WCG.

Proof. By Corollary $3, E^{*}$ admits an $M$-basis $\left\{\left(f_{i}, \hat{x}_{i}\right): i \in I\right\}$ with coefficient space $\operatorname{sp}\left\{\hat{x}_{i}\right\}=E$. Thus $\left\{\left(x_{i}, f_{i}\right)\right\}$ is a shrinking $M$-basis for $E$, so $E$ is WCG (see Zizler and John (1974a), page 10).

This recovers a result of Vašak (1974), page 221.

\section{Proof of the main result}

Let $E$ admit an $M$-basis $\left\{\left(x_{i}, f_{i}\right): i \in I\right\}$ with coefficient space $Y$. Assume $\|x\|_{Y}=1$ for each $i \in I$, and let $K=\left\{x_{i}: i \in I\right\}$. Clearly, $K \cup\{0\}$ is $\sigma(E, Y)$ compact.

Let $X$ denote the $\|\cdot\|_{Y}$ completion of $E$. Since $E$ and $X$ have (essentially) the same dual, $K \cup\{0\}$ is $\sigma(X, Y)$ compact, as well. Now since $X$ is a Banach space, the $\sigma(X, Y)$ closed convex hull of $K \cup\{0\} \cup\{-K\}$ is also $\sigma(X, Y)$ compact (Dunford and Schwartz (1958), page 434). Denote this set by $K_{1}$, and the gauge of $K_{1}$ by $\|\cdot\| \cdot \|$. Since $K_{1}$ is $\sigma(X, Y)$ compact it is $\|\cdot\|_{Y}$ bounded and so it may be assumed that $\|x\|_{Y} \leqslant\|x\|$ for all $x \in X$.

The proof of the Main Result involves modifying a sequence of lemmas due to Amir and Lindenstrauss (1968). 
LEMMA 1. Let $E$ admit an $M$-basis with coefficient space $Y$, and $X$ be its $\left\|_{Y}\right\|_{Y}$ completion. Then, given $\varepsilon>0$, an integer $n>0, m$ elements $f_{1}, f_{2}, \ldots, f_{m}$ of $S(Y)$, and a finite dimensional subspace $B \subset X$, there is $a\|\cdot\|_{Y}$ separable subspace $C$ of $X$ containing $B$ such that for every subspace $Z$, with $B \subset Z \subset X$ and $\operatorname{dim} Z / B=n$, there is a linear operator $T: Z \rightarrow C$ with $\|T\|_{Y},\|T\| \leqslant 1+\varepsilon, T b=b$ for all $b \in B$, and $\left|f_{k}(z)-f_{k}(T z)\right| \leqslant \varepsilon\|z\|$ for every $z \in Z$ and each $k, 1 \leqslant k \leqslant m$.

ProOF. Proceed exactly as in Lemma 3 of Amir and Lindenstrauss (1968). Note that in this case $K_{1}$ has the (relative) $\sigma(X, Y)$ topology and so the operator $T$ is the homogeneous extension of a $\sigma(X, Y)$ pointwise cluster point of a net on the compact Hausdorff space $\left(2 K_{1}\right)^{K_{1}}$.

LeMMA 2. (Amir and Lindenstrauss (1968, page 43) Let $E$ and $X$ be as in Lemma 1, $\mathscr{M}$ an infinite cardinal number, $G$ a subspace of $X$ with $\|\cdot\|_{Y}$ dens $G \leqslant \mathscr{M}$, and $F$ a subspace of $Y$ with $\sigma(Y, X)$ dens $F \leqslant \mathscr{M}$. Then there is a projection $P$ on $X$ with the following properties:

(1) $\|P\|_{Y}=\|P\|=1$;

(2) $P g=g$ for all $g \in G$;

(3) $P^{*} f=f$ for all $f \in F$; and

(4) $\|\cdot\|_{Y}$ dens $P X \leqslant \mathscr{M}$.

LEMMA 3. (Amir and Lindenstrauss (1968), page 44) Let $E$ and $X$ be as in Lemma 1 , and $\mu$ be the first ordinal of cardinality $\|\cdot\|_{Y}$ dens $X$. Then there is a transfinite sequence of projections $\left\{P_{\alpha}: \omega \leqslant \alpha \leqslant \mu\right\}$ on $X$ such that

(1) $\left\|P_{\alpha}\right\|_{Y}=\left\|P_{\alpha}\right\|=1$ for each $\alpha$;

(2) $\|\cdot\|_{Y}$ dens $P_{\alpha} X \leqslant$ cardinality of $\alpha$, for each $\alpha$;

(3) $P_{\alpha} P_{\beta}=P_{\beta} P_{\alpha}=P_{\beta}$ whenever $\beta<\alpha$; and

(4) $\bigcup_{\beta<\alpha} P_{\beta+1} X$ is $\|\cdot\|_{Y}$ dense in $P_{\alpha} X$ for every $\alpha>\omega$.

This next lemma is due to Troyanski (1971), page 175.

LEMMA 4. Let $E$ be a Banach space which satisfies the following properties:

(A) There is a continuous one-to-one linear operator $T$ which maps $E$ into $c_{0}(\Gamma)$, for some set $\Gamma$.

(B) There is a transfinite sequence of bounded linear operators $\left\{T_{\delta}: \delta \in D\right\}$ on $E$ such that

(1) for each $x \in E$ and each $\varepsilon>0$, the set

$$
\Lambda(x, \varepsilon)=\left\{\delta:\left\|T_{a+1} x-T_{a} x\right\|>\varepsilon\left(\left\|T_{a+1}\right\|+\left\|T_{\alpha}\right\|\right)\right.
$$

is finite;

(2) for each $x \in E, x \in \overline{\operatorname{sp}}\left[\left(\left\|T_{1} x\right\| T_{1} E\right) \cup \bigcup_{\delta \in \Lambda(x)}\left(T_{\alpha+1}-T_{\alpha}\right) E\right]$,

$$
\text { where } \Lambda(x)=\bigcup_{e>0} \Lambda(x, \varepsilon) \text {, }
$$


(3) dens sp $\left[\left(T_{\alpha+1}-T_{\alpha}\right) E\right] \leqslant$ dens $T_{1} E=\mathbf{N}_{0}$.

Then $E$ can be equivalently renormed to locally uniformly rotund.

In order to use Lemma 4 in the proof of the Main Result, one more lemma is needed.

Lemma 5. Let $X$ admit an $M$-basis $\left\{\left(x_{i}, f_{i}\right): i \in I\right\}$. Then $\left(P_{\alpha+1}-P_{a}\right) X$ admits an $M$-basis for each $\alpha, \omega \leqslant \alpha<\mu$.

ProOF Let $x_{i}^{\alpha}=\left(P_{\alpha+1}-P_{\alpha}\right) x_{i}$ and $f_{i}^{\alpha}=j_{\alpha}^{*} f_{i}$, where $j_{\alpha}^{*}$ is the mapping which restricts each $f_{i}$ to $\left(P_{\alpha+1}-P_{\alpha}\right) X$. After deleting all $x_{i}^{\alpha}$ and $f_{i}^{\alpha}$ which are zero, it must be shown that $\left\{\left(x_{j}^{\alpha}, f_{i}^{\alpha}\right): i \in I_{\alpha}\right\}$ is an $M$-basis for $\left(P_{a+1}-P_{\alpha}\right) X$. Firstly, $f_{j}^{\alpha}\left(x_{i}^{\alpha}\right)=f_{j}^{\alpha}\left(P_{\alpha+1}-P_{\alpha}\right) x_{i}=\left(P_{\alpha+1}-P_{\alpha}\right)^{*} f_{i}^{\alpha}\left(x_{i}\right)=f_{j}\left(x_{i}\right)$, since $\left(P_{\alpha+1}-P_{\alpha}\right)^{*}$ is the inverse of $j_{\alpha}^{*}$ for each $\alpha$. Thus if $\left\{\left(x_{i}, f_{i}\right)\right\}$ is biorthogonal, so is $\left\{\left(x_{i}^{\alpha}, f_{i}^{\alpha}\right)\right\}$ foreach $\alpha$. Next, for each $0 \neq y \in P_{\alpha+1} X$ there is an $x \in X \backslash\{0\}$ such that $P_{\alpha+1} x=y$, and so if $\left\{x_{i}\right\}$ is fundamental in $X$, then $\left(x_{i}^{\alpha}\right\}$ must be fundamental in $\left(P_{\alpha+1}-P_{a}\right) X$. Finally, if $x \in\left(P_{\alpha+1}-P_{\alpha}\right) X$ and $f_{i}^{\alpha}(x)=0$ for all $i \in I$, then $j_{\alpha}^{*} f_{i}(x)=0$ for all $i$. But this can not happen since $\left\{f_{i}\right\}$ is total over $X$. Thus $\left.\left\{x_{i}^{\alpha}, f_{i}^{\alpha}\right): i \in I\right\}$ is an $M$-basis for $\left(P_{\alpha+1}-P_{\alpha}\right) X$ for each $\alpha, \omega \leqslant \alpha<\mu$.

Proof of The Main Result The aim is to show that $X$ satisfies the conditions of Lemma 4. Since $E$ is $\|\cdot\|_{Y}$ dense in $X$, it is clear that if $E$ admits an $M$-basis, then this biorthogonal set is also an $M$-basis for $X$. By a result of Dyer (1969), page 55, if $X$ admits an $M$-basis, then $X$ satisfies condition (A) of Lemma 4 . Therefore it remains to construct a set of operators $\left\{T_{\delta}: \delta \in D\right\}$ on $E$ which satisfies (B1), (B2) and (B3).

If $X$ is separable, then $T_{\delta}=I$, the identity operator on $X$, for each $\delta \in D$. Therefore assume that $X$ is non-separable and proceed by transfinite induction on dens $X$.

Assume that the Main Result is true for all cardinal numbers less than dens $X$. By Lemma $3, X$ admits a transfinite sequence of projections $\left\{P_{\alpha}: \omega \leqslant \alpha \leqslant \mu\right\}$, where $\mu$ is the first ordinal number of cardinality dens $X$ and, by Lemma 5, each $\left(P_{\alpha+1}-P_{\alpha}\right) X$ also admits an $M$-basis. Hence, by the inductive hypothesis, there is a transfinite sequence of linear operators $\left\{S_{\beta}^{\alpha}: \omega \leqslant \beta \leqslant \Gamma_{\alpha}\right\}$, where $\Gamma_{\alpha}$ is the first ordinal of cardinality dens $\left(P_{\alpha+1}-P_{\alpha}\right) X$, which maps $\left(P_{\alpha+1}-P_{\alpha}\right) X$ into itself and satisfies (B1), (B2) and (B3) of Lemma 4. Let $\Lambda_{\alpha}$ denote the set of ordinal numbers $\gamma$, with $\omega \leqslant \gamma \leqslant \Gamma_{\alpha}$, and $D$ the set of ordered pairs of ordinal numbers $(\alpha, \beta)$, where $\beta \in \Lambda_{\alpha} \cup\{0\}$ and $\omega \leqslant \alpha \leqslant \mu$. Order this set lexiographically. For each $\delta=(\alpha, \beta) \in D$ define

$$
T_{\delta}= \begin{cases}S_{\beta}^{\alpha}\left(P_{\alpha+1}-P_{\alpha}\right)+P_{\alpha} & \text { if } \alpha<\mu \quad\left(S_{0}^{\alpha}=0\right), \\ P_{\mu}=I & \text { if } \alpha=\mu .\end{cases}
$$


As in Troyanski (1971), pages $177-178$, the set $\left\{T_{\delta}: \delta \in D\right\}$ satisfies the conditions (B1), (B2) and (B3) of Lemma 4. By Lemma 3, the operators $P_{\alpha}$ and $S_{\beta}^{\alpha}$ are $\sigma(E, Y)$ continuous on $X$ for each $\alpha, \omega \leqslant \alpha \leqslant \mu$, and each $\beta \in \Lambda_{\alpha}$. Thus, by Lemma $4, X$ can be renormed with an LUR norm which is $\sigma(X, Y)$ lower semi-continuous. Now restrict this norm to $E$ to get the Main Result.

THEOREM. Let $E$ admit an $M$-basis with a 1-norming coefficient space $Y$. If $\|\cdot\|_{Y}$ is a very smooth norm, then $E$ admits a shrinking $M$-basis, and so $E$ is WCG.

Proof. The fact that $Y$ is norming gives $E=X$, so proceed by transfinite induction on dens $E$. If $E$ is separable the result follows by applying Theorem III.1 of Johnson (1970b). Now assume the result is true for all cardinal numbers less than dens $E$. By Lemma 3 there is a transfintie sequence of projections $\left\{P_{\alpha}: \omega \leqslant \alpha \leqslant \mu\right\}$ defined on $E$ with dens $\left(P_{\alpha+1}-P_{\alpha}\right) E<\mu$ for all $\alpha, \omega \leqslant \alpha<\mu$, where $\mu$ is the first ordinal number of cardinality dens $E$. Since the coefficient space is 1-norming, $\|\cdot\|=\|\cdot\|_{2}$, so $E$ has a very smooth norm. Hence, by Tacon (1970) and Zizler and John (1974), page 3, there is a transfinite sequence of projections $\left\{P_{\alpha}^{*}: \omega \leqslant \alpha \leqslant \mu\right\}$ on $E^{*}$ which are continuous when $E^{*}$ has the weak-* topology. As in Tacon (1970), page 419, each $\left(P_{\alpha} E\right)^{*}$ may be identified (isometrically isomorphically) with $P^{*} E^{*}$. Now continue with the inductive procedure.

Since dens $\left(P_{\alpha+1}-P_{\alpha}\right) E<\mu$, the inductive hypothesis gives that $\left(P_{\alpha+1}-P_{\alpha}\right) E$ admits a shrinking $M$-basis $\left\{\left(x_{i}^{\alpha}, f_{i}^{\alpha}\right): i \in I_{\alpha}\right\}$ for each $\alpha, \omega \leqslant \alpha<\mu$. Since $\left(P_{\alpha+1}-P_{\alpha}\right)$ maps $E$ onto $\left(P_{\alpha+1}-P_{\alpha}\right) E$, the operator $\left(P_{\alpha+1}-P_{\alpha}\right)^{*}$ maps $\left[\left(P_{\alpha+1}-P_{\alpha}\right) E\right]^{*}$ isomorphically onto $\left(P_{\alpha+1}^{*}-P_{\alpha}^{*}\right) E^{*}$. Let $f_{i}^{\alpha}=\left(P_{\alpha+1}^{*}-P_{\alpha}^{*}\right) \tilde{f}_{i}^{\alpha}$ for each $i \in I$ and each $\alpha, \omega \leqslant \alpha<\mu$. Now it must be shown that $\left\{\left(x_{i}^{\alpha}, f_{i}^{\alpha}\right): i \in I_{\alpha}, \omega \leqslant \alpha<\mu\right\}$ is a shrinking $M$-basis for $E$.

Clearly, $\left\{x_{i}^{\alpha}\right\}$ is fundamental in $E$ since, by Lemma 3,

$$
\overline{\operatorname{sp}}\left\{x_{i}^{\alpha}\right\}=\bigcup_{\alpha<\mu}\left(P_{\alpha+1}-P_{\alpha}\right) E=E .
$$

Also,

$$
\overline{\operatorname{sp}}\left\{f_{i}^{\alpha}\right\}=\bigcup_{\alpha<\mu}\left(P_{\alpha+1}^{*}-P_{\alpha}^{*}\right) E^{*}=E^{*},
$$

since $\mathrm{E}$ is very smooth (Tacon (1970), page 421 ). The set $\left\{f_{i}^{\alpha}\right\}$ is total over $E$, since $\left\{f_{\alpha}^{i}\right\}$ is total over $\left(P_{\alpha+1}-P_{\alpha}\right) E$ for each $\alpha, \omega \leqslant \alpha<\mu$. Therefore, it only remains to show that $\left\{\left(x_{i}^{\alpha}, f_{i}^{\alpha}\right)\right\}$ is a biorthogonal set.

If $\alpha \neq \beta$, then

$$
\begin{aligned}
f_{i}^{\alpha}\left(x_{i}^{\beta}\right) & =\left(P_{\alpha+1}^{*}-P_{\alpha}^{*}\right) f_{i}^{\alpha}\left(x_{i}^{\beta}\right) \\
& =\left(P_{\alpha+1}^{*}-P_{\alpha}^{*}\right)\left(\tilde{f}_{i}^{\alpha}\right)\left(P_{\beta+1}-P_{\beta}\right)\left(x_{i}^{\beta}\right) \\
& =\tilde{f}_{i}^{\alpha}\left(P_{\alpha+1}-P_{\alpha}\right)\left(P_{\beta+1}-P_{\beta}\right) x_{i}^{\beta} .
\end{aligned}
$$


But, by Lemma $3, P_{\alpha} P_{\beta}=P_{\beta} P_{\alpha}=P_{\beta}$ whenever $\beta<\alpha$ and so

$$
\left(P_{\alpha+1}-P_{\alpha}\right)\left(P_{\beta+1}-P_{\beta}\right)=0 \text {. }
$$

Hence $f_{i}^{\alpha}\left(x_{i}^{\beta}\right)=0$ for all $x_{i}^{\beta}$ with $\beta \neq \alpha$. By construction

$f_{i}^{\alpha}\left(x_{j}^{\alpha}\right)=1$ if $i=j$, and $f_{i}^{\alpha}\left(x_{j}^{\alpha}\right)=0$ otherwise. Therefore $\left\{x_{i}^{\alpha}, f_{j}^{\alpha}\right\}$ is biorthogonal, and this completes the proof.

\section{References}

D. Amir and J. Lindenstrauss (1968), 'The structure of weakly compact sets in Banach spaces' Ann. of Math. (2) 88, 35-46.

E. Asplund (1967), 'Averaged norms', Israel J. Math. 5, 227-233.

N. Dunford and J. Schwartz (1958), Linear operators, Part I (Interscience, New York).

J. Dyer (1968), 'Generalized Markuschevich bases', Israel J. Math. 7, 51-59.

J. R. Giles (1971), 'On a characterization of the differentiability of the norm of a normed linear space', J. Austral. Math. Soc. Ser. A 12, 106-114.

J. R. Giles (1975), 'On smoothness of the Banach space embedding', Bull. Austral. Math. Soc. 13, 69-74.

W. B. Johnson (1970a), 'Markuschevich bases and duality theory', Trans. Amer. Math. Soc. 149, 171-177.

W. B. Johnson (1970b), 'No infinite dimensional $P$ space admits a Markuschevich basis', Proc. Amer. Math. Soc. 28, 467-468.

V. Klee (1953), 'Convex bodies and periodic homeomorphisms in Hilbert spaces', Trans. Amer. Math. Soc. 74, 10-43.

J. Lindenstrauss (1972), 'Weakly compact sets-their topological properties and the Banach spaces they generate', Ann. of Math. Studies 69, 235-273.

A. Lovaglia (1955), 'Locally uniformly convex Banach spaces', Trans. Amer. Math. Soc. 78, 225-238.

D. C. Tacon (1970), 'The conjugate of a smooth Banach space', Bull. Austral. Math. Soc. 2, 415425.

S. Troyanski (1971), 'On locally uniformly convex and differentiable norms in certain non-spearable Banach spaces', Studia Math. 37, 173-180.

L. Vašak (1973), 'Generalized Markuschevich bases', Comm. Math. Univ. Carol. 14, 92, 215-222.

V. Zizler and K. John (1974a), 'Smoothness and its equivalents in weakly compactly generated spaces', J. Functional Analysis 15, 1-11.

V. Zizler and K. John (1974b), 'Some remarks on non-separable Banach spaces with Markuschevich bases', Comm. Math. Univ. Carol.15 (4), 679-691.

Department of Mathematics

University of New England

Armidale, N.S.W. 2351

Australia 